\title{
SUBGROUPS OF DIRECT PRODUCTS OF TWO LIMIT GROUPS
}

\author{
Martin R. Bridson and James Howie
}

\begin{abstract}
If $\Gamma_{1}$ and $\Gamma_{2}$ are limit groups and $S \subset \Gamma_{1} \times \Gamma_{2}$ is of type $\mathrm{FP}_{2}$ then $S$ has a subgroup of finite index that is a product of at most two limit groups.
\end{abstract}

\section{Introduction}

In [6] Miller, Short and the present authors described the homological finiteness properties of subgroups of direct products of free and surface groups. This completed a programme of research that began with celebrated examples of Stallings [24] and Bieri [5] and took an unexpected turn when Baumslag and Roseblade [3] showed that the only finitely presented subdirect ${ }^{1}$ products of two free groups are the obvious ones, namely free groups and subgroups of finite index.

This last theorem admits instructive proofs from several perspectives [9], [23], [16]; each of these proofs lends new insight and leads to an interesting generalisation, but each relies heavily on the fact that one is dealing with only two factors. The search for a higher-dimensional analogue culminated in the Main Theorem of [6], which shows in particular that if $S$ is a subgroup of a direct product of $n$ free or surface groups and the homology of $S$ (with trivial coefficients) is finitely generated up to dimension $n$, then $S$ has a subgroup of finite index that is isomorphic to a direct product of free or surface groups.

Recent work related to the Tarski conjectures ([13] to [15]; [20] to [22]) has created great interest in the class of groups called fully residually free groups in [2] and $\omega$ residually free groups in [17]. Nowadays, the groups in this class are commonly referred to as limit groups. Remeslennikov [17] proved that these are the finitely generated groups that have the same existential theory as free groups. Sela [19] has proposed a list of central questions concerning limit groups. In particular he asked whether the results of [6] could be extended to subdirect products of limit groups. In the first two papers in this series [7], [8], we established results concerning the subgroup structure of limit groups that enabled us to answer this question in the case where the limit groups have the same elementary theory as a free group. In the present article we turn our attention to the subdirect products of general limit groups. We use our previous results in tandem with calculations in group homology and geometrical arguments based on Bass-Serre theory to prove the following analogue of the Baumslag-Roseblade theorem.

Received by the editors November 15, 2005.

2000 Mathematics Subject Classification. 20F65, 20E08, $20 \mathrm{~F} 67$.

Key words and phrases. limit groups, finiteness properties, Bass-Serre theory.

${ }^{1}$ Recall that $S \subset A \times B$ is termed a subdirect product if its projection to both $A$ and $B$ is surjective. 
Theorem 1.1. Let $S \subset \Gamma_{1} \times \Gamma_{2}$ be a subdirect product of non-abelian limit groups. If $S$ is of type $\mathrm{FP}_{2}(\mathbb{Q})$ and $L_{i}=S \cap \Gamma_{i}$ is non-trivial for $i=1,2$, then $S$ has finite index in $\Gamma_{1} \times \Gamma_{2}$.

Some simple reductions (4.1) yield:

Corollary 1.2. Let $\Gamma_{1}$ and $\Gamma_{2}$ be limit groups. If $S \subset \Gamma_{1} \times \Gamma_{2}$ is finitely presented then $S$ has a subgroup of finite index that is a product of at most two limit groups.

This is a special case of the following result, which is proved in section 5 .

Corollary 1.3. Let $\Gamma_{1}, \ldots, \Gamma_{n}$ be limit groups, with $\Gamma_{3}, \ldots, \Gamma_{n}$ abelian, and let $S \subset$ $\Gamma_{1} \times \cdots \times \Gamma_{n}$ be a subdirect product such that $S$ is of type $\mathrm{FP}_{m}(\mathbb{Q})$, where $m=$ $\min (n, 3)$. Then $S$ is isomorphic to a subgroup of finite index in a product of at most $m$ limit groups.

For simplicity of exposition, the homology of a group $G$ in this paper will always be with coefficients in a $\mathbb{Q} G$-module - typically the trivial module $\mathbb{Q}$. With minor modifications, our arguments also apply with other coefficient modules, giving corresponding results under the finiteness conditions $\mathrm{FP}_{n}(R)$ for other suitable rings $R$.

The final section of this paper contains some speculation concerning the subgroups of the direct product of arbitrarily many limit groups.

\section{Limit groups, their decomposition and subgroup structure}

Since this is the third in a series of papers (following $[7,8]$ ), we shall only recall the minimal necessary amount of information about limit groups. The reader unfamiliar with this fascinating class of groups can consult the seminal papers of Sela [20] to [22] or the excellent introduction [4]. The papers of Kharlampovich and Myasnikov $[13,14]$ approach the subject from a perspective more in keeping with traditional combinatorial group theory, and a further perspective is developed in [11].

2.1. Limit groups. A finitely generated group $\Gamma$ is fully residually free if for every finite set $T \subset \Gamma$ there exists a homomorphism $\phi$ from $\Gamma$ to a free group so that $\left.\phi\right|_{T}$ is injective. This condition is known to be equivalent to Sela's definition of a limit group [20]. In the sequel, we will exclusively use the term limit group.

Our results rely on the fact that limit groups are the finitely generated subgroups of $\omega$-residually free tower $(\omega$-rft) groups $[21,13]$. Such tower groups are the fundamental groups of tower spaces that are assembled from graphs, tori and surfaces in a hierarchical manner. The number of stages in the process of assembly is the height of the tower. Each stage in the construction involves the attachment of a surface along its boundary, or the attachment of an $n$-torus $T$ along an embedded circle representing a primitive element of $\pi_{1} T$.

The height of a limit group $S$ is the minimal height of an $\omega$-rft group that has a subgroup isomorphic to $S$. Limit groups of height 0 are free products of finitely many free abelian groups (each of finite rank) and surface groups of Euler characteristic at most -2 .

The splitting described in the following proposition is obtained as follows: embed $\Gamma$ in an $\omega$-rft group $G$, take the graph of groups decomposition that the Seifert-van 
Kampen Theorem associates to the addition of the final block in the tower, then apply Bass-Serre theory to get an induced graph of groups decomposition of $\Gamma$.

Proposition 2.1. If $\Gamma$ is a limit group of height $h \geq 1$, then $\Gamma$ is the fundamental group of a finite graph of groups with the following properties:

(1) the vertex groups are limit groups of height $\leq h-1$;

(2) the edge groups are trivial or infinite-cyclic;

(3) each edge is adjacent to a vertex where the vertex-group is non-abelian.

Note also that any non-abelian limit group of height 0 splits as $A *_{C} B$ with $C$ infinite-cyclic or trivial.

2.2. Homological finiteness implies finiteness. The following is proved in [7].

Theorem 2.2. Let $\Gamma$ be a limit group and $S \subset \Gamma$ a subgroup. If $H_{1}(S, \mathbb{Q})$ is finite dimensional then $S$ is finitely generated (and hence is a limit group).

2.3. Normal subgroups. From [7] we need:

Theorem 2.3. If $\Gamma$ is a limit group and $H \subset \Gamma$ is a finitely generated subgroup, then either $H$ has finite index in its normaliser or else the normaliser of $H$ is abelian.

And from [8] we need:

Theorem 2.4. Let $\Gamma$ be a limit group that splits as an amalgamated free product or $H N N$ extension with cyclic edge group $C \subset \Gamma$.

If $N \subset \Gamma$ is a non-trivial normal subgroup then there exists a subgroup $\Gamma_{0} \subset \Gamma$ of finite index and an element $t \in N \cap \Gamma_{0}$ such that $\Gamma_{0}$ is an $H N N$-extension with stable letter $t$ and amalgamated subgroup $C \cap \Gamma_{0}$.

2.4. Double cosets. We also require the following Double Coset Lemma from [8].

Theorem 2.5. Suppose that, for $i=1, \ldots, n, \Gamma_{i} * A_{i}$ is an $H N N$ extension with associated subgroup $A_{i}$ and stable letter $t_{i}$. Let $\Gamma=\Gamma_{1} \times \cdots \times \Gamma_{n}, A=A_{1} \times \cdots \times A_{n}$, and let $S \subset \Gamma$ be a subgroup containing $t_{1}, \ldots, t_{n}$. Then $H_{n}(S, \mathbb{Q})$ contains a subgroup isomorphic to

$$
\mathbb{Q} \otimes_{\mathbb{Q} S} \mathbb{Q} \Gamma \otimes_{\mathbb{Q} A} \mathbb{Q}
$$

a rational vector space with basis the set $S \backslash \Gamma / A$ of double cosets $S g A$ with $g \in \Gamma$.

In the proof of Theorem 1.1 we will need the above result in the case $n=2$. In this case, if $H_{2}(S, \mathbb{Q})$ is finite dimensional then $|S \backslash \Gamma / A|<\infty$. Thus it is of interest to understand when a pair of subgroups in a group admit only finitely many double cosets. The following assertions are proved in [8], and are easily verified.

Proposition 2.6. Let $G$ be a group, $A, B, C$ subgroups of $G$ with $A \subset C$ and $|B \backslash G / A|<\infty$, and $f: G \rightarrow H$ a homomorphism. Then

(1) $|(B \cap C) \backslash C / A|<\infty$, and

(2) $|f(B) \backslash f(G) / f(A)|<\infty$.

\section{Some homological algebra}

In this section we gather the homological algebra that we shall need in the proof of the main theorem. 
3.1. The LHS spectral sequence. Associated to any short exact sequence of groups $1 \rightarrow A \rightarrow B \rightarrow C \rightarrow 1$ one has the Lyndon-Hochschild-Serre (LHS) spectral sequence in homology (see [10, p. 171], or [12, p.303]). Given a $\mathbb{Q} B$-module $M$, the $E^{2}$ page of the spectral sequence has $(p, q)$-entry $H_{p}\left(C, H_{q}(A, M)\right)$, and the spectral sequence converges to $H_{*}(B, M)$.

The computations in this article concern $H_{i}(B)$ with trivial coefficients and $i \leq 2$. The terms of relevance on the $E^{2}$ page are

$$
H_{0}\left(C, H_{2}(A)\right), H_{1}\left(C, H_{1}(A)\right), H_{2}\left(C, H_{0}(A)\right) \text {. }
$$

In each of our calculations, the first and last of these terms will be finitely generated and we will be concerned with the issue of finite generation for $H_{1}\left(C, H_{1}(A)\right)$. The only non-zero derivative hitting this group is $d_{2}: H_{3}\left(C, H_{0}(A)\right) \rightarrow H_{1}\left(C, H_{1}(A)\right)$. Since the terms $E_{p, q}^{\infty}$ with $p+q=2$ are the composition factors of a filtration of $H_{2}(B)$, we see that $H_{2}(B)$ will be finitely generated only if the quotient of $H_{1}\left(C, H_{1}(A)\right)$ by the image of $d_{2}$ is finitely generated. But $H_{3}\left(C, H_{0}(A)\right)$ will always be finitely generated in our setting, and hence $H_{2}(B)$ will be finitely generated only if $H_{1}\left(C, H_{1}(A)\right)$ is finitely generated.

3.2. Fox calculus. Recall that a presentation $\langle X \mid R\rangle$ of a group $G$ gives rise to a partial free $\mathbb{Q} G$-resolution

$$
F_{2} \stackrel{\partial_{2}}{\rightarrow} F_{1} \stackrel{\partial_{1}}{\rightarrow} \mathbb{Q} G \rightarrow \mathbb{Q}
$$

of the trivial $G$-module $\mathbb{Q}$. Here $F_{1}$ and $F_{2}$ are free modules with bases in one-to-one correspondence with $X$ and $R$ respectively.

The boundary maps $\partial_{1}$ and $\partial_{2}$ are easy to calculate. $\partial_{1}$ is given by an $X \times 1$-matrix where the $x$-entry is $1-x$, and $\partial_{2}$ is given by an $R \times X$-matrix where the $(r, x)$-entry is the Fox derivative $\partial r / \partial x$, defined inductively on the length of $r$ by the rules:

$$
\begin{gathered}
\frac{\partial}{\partial x}(1)=0=\frac{\partial}{\partial x}(y)=\frac{\partial}{\partial x}\left(y^{-1}\right), y \in X \backslash\{x\}, \\
\frac{\partial}{\partial x}(x)=1, \frac{\partial}{\partial x}\left(x^{-1}\right)=-x^{-1}, \frac{\partial}{\partial x}(u v)=\frac{\partial u}{\partial x}+u \frac{\partial v}{\partial x} .
\end{gathered}
$$

Lemma 3.1. Let $\Gamma=G *_{C}$ be an $H N N$ extension with stable letter $t$, and let $M$ be a $\mathbb{Q} \Gamma$-module on which $t$ acts trivially. Then $H_{1}(\Gamma, M)$ contains a subgroup that admits an epimorphism onto $H_{0}(C, M)$.

Proof. Given a presentation $\langle X \mid R\rangle$ of $G$, and a set of generators $Y$ for $C$ (expressed as words in the alphabet $X)$, we have a presentation for $\Gamma$ of the form

$$
\mathcal{P}=\left\langle X, t \mid R, y t u_{y} t^{-1}(y \in Y)\right\rangle
$$

for $\Gamma$ (where the $u_{y}$ are words in $X$ ).

We calculate $H_{1}(\Gamma, M)$ by tensoring the resolution associated to this presentation with $M$ and calculating $\partial_{2}$ using Fox calculus. Specifically, we have

$$
\bigoplus_{y \in Y} M \oplus \bigoplus_{r \in R} M \stackrel{\partial_{2}}{\rightarrow} M \oplus \bigoplus_{x \in X} M \stackrel{\partial_{1}}{\rightarrow} M \rightarrow 0
$$

With regard to the visible decompositions, $\partial_{2}$ is given by a matrix of the form

$$
\left(\begin{array}{cc}
\frac{\partial}{\partial t}\left(y t u_{y} t^{-1}\right) & * \\
0 & *
\end{array}\right),
$$


where $\frac{\partial}{\partial t}\left(y t u_{y} t^{-1}\right)=y-1$, while the restriction of $\partial_{1}$ to the first factor is multiplication by $(1-t)$. But since $t$ acts trivially on $M$, multiplication by $(1-t)$ is the zero-map on $M$. Therefore the first factor $M$ in dimension 1 is contained in the group of 1-cycles. Let $A$ denote the subgroup of $H_{1}(\Gamma, M)$ generated by this group of 1-cycles.

Now the first coordinate of the image of $\partial_{2}$ is contained in the submodule $N$ of $M$ generated by $\{(y-1) M, y \in Y\}$. It follows that the kernel of the natural epimorphism $M \rightarrow A$ is contained in $N$. Thus there is an epimorphism $A \rightarrow M / N$, and since $Y$ generates $C$, the quotient $M / N$ is the group of coinvariants $H_{0}(C, M)$.

3.3. A mapping cylinder argument. We are grateful to Peter Kropholler for a helpful conversation concerning the following lemma.

Lemma 3.2. If $G$ is of type $\mathrm{FP}_{n}(\mathbb{Q})$ and acts cocompactly on a tree with all edgestabilisers of type $\mathrm{FP}_{n}(\mathbb{Q})$, then all vertex-stabilisers are also of type $\mathrm{FP}_{n}(\mathbb{Q})$.

Proof. If $V, E$ are the vertex and edge sets of the tree, then the hypothesis on edge-stabilisers is equivalent to $\mathbb{Q} E$ having a projective $\mathbb{Q} G$-resolution that is finitely generated in dimensions $\leq n$, and the assertion of the lemma is that the analogous property for $\mathbb{Q} V$ holds.

Since the action is cocompact, $\mathbb{Q} V$ is finitely generated as a $\mathbb{Q} G$-module, so there is a projective $\mathbb{Q} G$-resolution $\mathcal{F}$ for $\mathbb{Q} V$ that is finitely generated in dimension 0 .

Suppose inductively that $k<n$ and there is a projective $\mathbb{Q} G$-resolution $\mathcal{F}$ for $\mathbb{Q} V$ that is finitely generated in dimensions $\leq k$. Let $\mathcal{P}$ be a projective $\mathbb{Q} G$-resolution for $\mathbb{Q} E$ that is finitely generated in dimensions $\leq n$.

Let $f: \mathcal{P} \rightarrow \mathcal{F}$ be a chain-map lifting the boundary map $\mathbb{Q} E \rightarrow \mathbb{Q} V$, and form the mapping cylinder resolution $\mathcal{M}=\mathcal{M}(f)$ of $\mathbb{Q}$.

Thus $\mathcal{M}_{i}=\mathcal{P}_{i-1} \oplus \mathcal{F}_{i}$ is finitely generated in dimensions $i \leq k$. The boundary maps $D_{i}: \mathcal{M}_{i} \rightarrow \mathcal{M}_{i-1}$ are defined by

$$
D_{i}(x, y):=\left(d^{\mathcal{P}}(x),(-1)^{i-1} f(x)+d^{\mathcal{F}}(y)\right),
$$

where $d^{\mathcal{P}}$ and $d^{\mathcal{F}}$ are the boundary maps in $\mathcal{P}$ and $\mathcal{F}$ respectively.

Since $k<n$ and $G$ is of type $\mathrm{FP}_{n}(\mathbb{Q})$, the kernel of the $k$-th boundary map $D_{k}: \mathcal{M}_{k} \rightarrow \mathcal{M}_{k-1}$ is finitely generated. Since also $\mathcal{P}_{k+1}$ is finitely generated, there is a finitely generated $\mathbb{Q} G$-direct summand $L$ of $F_{k+1}$ such that

(i) $D_{k+1}\left(\mathcal{P}_{k} \oplus L\right)=\operatorname{Ker}\left(D_{k}\right)=D_{k+1}\left(\mathcal{M}_{k+1}\right)$; and

(ii) $f_{k+1}\left(\mathcal{P}_{k+1}\right) \subset L$.

Now suppose that $a \in \operatorname{Ker}\left(d_{k}: \mathcal{F}_{k} \rightarrow \mathcal{F}_{k-1}\right)$. Then $(0, a) \in \operatorname{Ker}\left(D_{k}\right)$, so by (i) we can find $b \in \mathcal{P}_{k}$ and $c \in L$ such that

$$
(0, a)=D_{k+1}(b, c)=\left(d_{k}(b),(-1)^{k} f_{k}(b)+d_{k+1}(c)\right) .
$$

Then $b \in \operatorname{Ker}\left(d_{k}\right)=\operatorname{Im}\left(d_{k+1}\right)$, so we can find $e \in \mathcal{P}_{k+1}$ such that $b=d_{k+1}(e)$. Then $f_{k}(b)=f_{k}\left(d_{k+1}(e)\right)=d_{k+1}\left(f_{k+1}(e)\right)$, and

$$
a=(-1)^{k} f_{k}(b)+d_{k+1}(c)=d_{k+1}\left((-1)^{k} f_{k+1}(e)+c\right) \in d_{k+1}(L)
$$

(using (ii)).

Hence $\operatorname{Ker}\left(d_{k}: F_{k} \rightarrow F_{k-1}\right)=d_{k+1}(L)$ is finitely generated. It follows that there is a projective $\mathbb{Q} G$-resolution of $\mathbb{Q} V$ that is finitely generated in dimensions $\leq k+1$. 
By induction, there is a projective $\mathbb{Q} G$-resolution of $\mathbb{Q} V$ that is finitely generated in dimensions $\leq n$. In other words, the vertex stabilisers of the action are all of type $\mathrm{FP}_{n}(\mathbb{Q})$, as claimed.

\section{The main argument}

In this section we prove Theorem 1.1 and Corollary 1.2.

4.1. Reduction to subdirect products. Let $\Gamma_{1}$ and $\Gamma_{2}$ be two non-abelian limit groups. Let $S \subset \Gamma_{1} \times \Gamma_{2}$ be a subgroup and let $p_{i}: S \rightarrow \Gamma_{i}$ be the natural projection. If $S$ is of type $\mathrm{FP}_{2}(\mathbb{Q})$, then $H_{1}(S, \mathbb{Q})$ is finite dimensional, so $H_{1}\left(p_{i}(S)\right.$, $\left.\mathbb{Q}\right)$ is finite dimensional for $i=1,2$. By Theorem 2.2, $p_{i}(S) \subset \Gamma_{i}$ is a limit group. Replacing $\Gamma_{i}$ by $p_{i}(S)$, we reduce to the case where $S$ projects onto each factor $\Gamma_{i}$.

Let $L_{i}=S \cap \Gamma_{i}$. If $L_{1}$ were trivial, then $S \cong p_{2}(S)$ would be a limit group, satisfying the conclusion of Corollary 1.2. Thus we may assume that $L_{i}$ is non-trivial for $i=1,2$ (and Corollary 1.2 becomes a weak form of Theorem 1.1).

Remark 4.1. Since $p_{i}(S)=\Gamma_{i}$ and $L_{i}$ is normal in $S$, we also have that $L_{i}=p_{i}\left(L_{i}\right)$ is normal in $\Gamma_{i}$.

4.2. Subgroups of finite index. Throughout the proof we shall repeatedly pass to subgroups of finite index in one or other of the $\Gamma_{i}$. When we do so, we shall be assuming that $S$ is replaced with the inverse image of this subgroup under the projection $p_{i}: S \rightarrow \Gamma_{i}$ and $\Gamma_{j}(j \neq i)$ is replaced by $p_{j} p_{i}^{-1}\left(H_{i}\right)$. This does not affect $L_{j}$

Remark 4.2. It is because of the need to pass to subgroups of finite index that we have assumed that $S$ is of type $\mathrm{FP}_{2}$ rather than simply that $H_{2}(S)$ is finite dimensional: the former hypothesis passes to subgroups of finite index, whereas the latter does not necessarily.

4.3. Useful lemmas and special cases. Much of our proof will revolve around the LHS spectral sequences associated to the short exact sequences for the projections of $S$ to the two factors $1 \rightarrow L_{2} \rightarrow S \rightarrow \Gamma_{1} \rightarrow 1$ and $1 \rightarrow L_{1} \rightarrow S \rightarrow \Gamma_{2} \rightarrow 1$. In particular, following the discussion in subsection 3.1, we shall use the hypothesis that $S$ is of type $\mathrm{FP}_{2}$ by assuming that $H_{1}\left(\Gamma_{i}, H_{1}\left(L_{j}, \mathbb{Q}\right)\right)$ is finitely generated for $j \neq i$. In the ensuing argument we shall make several appeals to the following:

Lemma 4.3. Let $S$ be a subdirect product of two non-abelian limit groups $\Gamma_{1}$ and $\Gamma_{2}$, such that the intersections $L_{1}=S \cap \Gamma_{1}$ and $L_{2}=S \cap \Gamma_{2}$ are both non-trivial. If either $H_{1}\left(L_{1}, \mathbb{Q}\right)$ or $H_{1}\left(L_{2}, \mathbb{Q}\right)$ is finite dimensional, then $S$ has finite index in $\Gamma_{1} \times \Gamma_{2}$.

Proof. Suppose that $H_{1}\left(L_{1}, \mathbb{Q}\right)$ is finite dimensional. It follows from Theorem 2.2 that $L_{1}$ is finitely generated, and from Theorem 2.3 and Remark 4.1 that $L_{1}$ has finite index in $\Gamma_{1}$. Therefore, after replacing $\Gamma_{1}$ by $L_{1}$, we can split $1 \rightarrow L_{2} \rightarrow S \rightarrow \Gamma_{1} \rightarrow 1$ to obtain $S=\Gamma_{1} \times L_{2}$. Thus $L_{2}$, being a retract of $S$, is finitely generated, so of finite index in $\Gamma_{2}$.

Proposition 4.4. Let $G_{1}$ be a group and let $\Gamma_{2}$ be a limit group. Let $S \subset G_{1} \times \Gamma_{2}$ be a subgroup of type $\mathrm{FP}_{2}(\mathbb{Q})$ that projects onto $\Gamma_{2}$, and that intersects $\Gamma_{2}$ non-trivially. Suppose that $\Gamma_{2}$ can be expressed as an amalgamated free product or HNN extension with cyclic edge-group $C$.

If $C \cap S$ has finite index in $C$, then $H_{1}\left(G_{1} \cap S, \mathbb{Q}\right)$ is finite dimensional. 
Proof. Let $L_{1}=G_{1} \cap S$ and let $L_{2}=\Gamma_{2} \cap S$. Since the cyclic subgroups of $\Gamma_{2}$ are closed in the profinite topology, we may pass to a subgroup of finite index which intersects $C$ in $C \cap L_{2}$ and hence assume that $C \subset L_{2}$.

By appealing to Theorem 2.4 and passing to a further subgroup of finite index, we may assume that $\Gamma_{2}$ is an HNN extension $G *_{C}$ with stable letter $t \in L_{2}$.

Consider the Lyndon-Hochschild-Serre spectral sequence in $\mathbb{Q}$-homology corresponding to the group extension

$$
1 \rightarrow L_{1} \rightarrow S \rightarrow \Gamma_{2} \rightarrow 1 .
$$

The action of $S$ by conjugation on $L_{1}$ induces an action of $\Gamma_{2}=S / L_{1}$ on $H_{*}\left(L_{1}, \mathbb{Q}\right)$ in which $L_{2}$ acts trivially. In particular $t$ and $C$ act trivially on the $\Gamma_{2}$-module $H_{k}\left(L_{1}, \mathbb{Q}\right)$, for each $k$.

The $(1,1)$-term $E_{1,1}^{3}$ on the $E^{3}$-page of this spectral sequence survives to infinity, and so is isomorphic to a subquotient of $H_{2}(S, \mathbb{Q})$, which is finite-dimensional, by hypothesis. Hence $E_{1,1}^{3}$ is finite-dimensional. But $E_{1,1}^{3}$ is the cokernel of the differential $d: E_{3,0}^{2} \rightarrow E_{1,1}^{2}$ from the $E^{2}$-page. Moreover, $E_{3,0}^{2}=H_{3}\left(\Gamma_{2}, H_{0}\left(L_{1}, \mathbb{Q}\right)\right)=$ $H_{3}\left(\Gamma_{2}, \mathbb{Q}\right)$ is finite-dimensional, since the limit group $\Gamma_{2}$ has type $\mathrm{FP}_{\infty}(\mathbb{Q})$. Hence $E_{1,1}^{2}=H_{1}\left(\Gamma_{2}, H_{1}\left(L_{1}, \mathbb{Q}\right)\right)$ is finite-dimensional.

Since $t$ acts trivially on $H_{*}\left(L_{1}, \mathbb{Q}\right)$, we may apply Lemma 3.1, with $\Gamma=\Gamma_{2}$ and $M=H_{1}\left(L_{1}, \mathbb{Q}\right)$. It follows that $H_{0}\left(C, H_{1}\left(L_{1}, \mathbb{Q}\right)\right)$ is finite-dimensional. And $H_{0}\left(C, H_{1}\left(L_{1}, \mathbb{Q}\right)\right) \cong H_{1}\left(L_{1}, \mathbb{Q}\right)$ since $C$ acts trivially on $H_{*}\left(L_{1}, \mathbb{Q}\right)$.

The spectral sequence argument ending with the penultimate paragraph of the preceding proof admits a straightforward generalisation as follows. Suppose that $S$ is of type $\mathrm{FP}_{n}, 1 \leq k \leq n-1$ and that $H_{j}\left(L_{1}, \mathbb{Q}\right)$ is finite dimensional for each $j<k$. Then $E_{1, k}^{2}=H_{1}\left(\Gamma_{2}, H_{k}\left(L_{1}, \mathbb{Q}\right)\right)$ is finite-dimensional. Taking $M=H_{k}\left(L_{1}, \mathbb{Q}\right)$ in the final paragraph then implies $H_{k}\left(L_{1}, \mathbb{Q}\right) \cong H_{0}\left(C, H_{k}\left(L_{1}, \mathbb{Q}\right)\right)$ is finite-dimensional.

To generalise further, recall that Theorem 2.4 is a weak form of what we actually proved in [8] concerning the existence of stable letters in normal subgroups. Correspondingly, we can weaken the hypotheses on $\Gamma_{2}$ and $C$ in the above proposition, and appeal to [8, Corollaries 2.2 and 3.2] instead of Theorem 2.4. These remarks lead to the following generalisation (which will not be needed in the sequel).

Proposition 4.5. Let $G_{1}$ and $G_{2}$ be groups. Suppose $G_{2}$ is of type $\mathrm{FP}_{n+1}(\mathbb{Q})$ and admits an l-acylindrical ${ }^{2}$ splitting (for some $l>0$ ) over a subgroup $C$ that is closed in the profinite topology.

Let $S \subset G_{1} \times G_{2}$ be a subgroup of type $\mathrm{FP}_{n}$ that intersects $G_{2}$ non-trivially and intersects $C$ in a subgroup of finite index. Then $H_{i}\left(G_{1} \cap S, \mathbb{Q}\right)$ is finite dimensional for $i \leq n-1$.

In the light of Lemma 4.3, the following is an immediate consequence of Proposition 4.4 .

Corollary 4.6. Let $\Gamma_{1}$ and $\Gamma_{2}$ be non-abelian limit groups. Let $S \subset \Gamma_{1} \times \Gamma_{2}$ be a subdirect product of type $\mathrm{FP}_{2}(\mathbb{Q})$ that intersects each of the factors non-trivially. Suppose that $\Gamma_{2}$ has a graph-of-groups decomposition with a cyclic edge-group $C$.

If $C \cap S$ has finite index in $C$, then $S$ has finite index in $\Gamma_{1} \times \Gamma_{2}$.

\footnotetext{
${ }^{2} \mathrm{~A}$ splitting is $l$-acylindrical if arcs of length $l$ in the associated Bass-Serre tree have trivial stabilizers.
} 
In the case where $C$ is trivial we have:

Corollary 4.7. Let $\Gamma_{1}$ and $\Gamma_{2}$ be non-abelian limit groups and let $S \subset \Gamma_{1} \times \Gamma_{2}$ be a subdirect product of type $\mathrm{FP}_{2}(\mathbb{Q})$ that intersects each of the factors non-trivially. If $\Gamma_{2}$ is a nontrivial free product, then $S$ has finite index in $\Gamma_{1} \times \Gamma_{2}$.

The remaining non-abelian limit groups of height 0 are covered by:

Corollary 4.8. Let $\Gamma_{1}$ and $\Gamma_{2}$ be non-abelian limit groups with $\Gamma_{2}$ the fundamental group of a compact surface. Let $S \subset \Gamma_{1} \times \Gamma_{2}$ be a subdirect product that intersects each of the factors non-trivially.

If $S$ is of type $\mathrm{FP}_{2}(\mathbb{Q})$, then $S$ has finite index in $\Gamma_{1} \times \Gamma_{2}$.

Proof. Passing to an index 2 subgroup of $\Gamma_{2}$ if necessary, we may assume that the surface in question is orientable, and by Corollary 4.7 we may assume that it is closed.

Fix $\alpha \neq 1$ in $L_{2}$. By a theorem of Scott [18], there is a finite-sheeted cover of $\Sigma$ in which $\alpha$ lifts to a simple closed curve. Replacing $\Gamma_{2}$ by the corresponding subgroup of finite index, we may assume that $\Gamma_{2}$ splits over the cyclic subgroup $\langle\alpha\rangle \subset L_{2}$. The result then follows from Corollary 4.6.

The following observation will be used to control the way in which $S$ intersects each non-abelian vertex group in the decomposition of $\Gamma_{2}$ provided by Proposition 2.1 .

Lemma 4.9. Let $\Gamma_{1}$ and $\Gamma_{2}$ be non-abelian limit groups and $S \subset \Gamma_{1} \times \Gamma_{2}$ a subdirect product of type $\mathrm{FP}_{2}(\mathbb{Q})$ with $L_{i}=\Gamma_{i} \cap S$ non-trivial for $i=1,2$. Consider a graph-ofgroups decomposition $\mathcal{G}$ of $\Gamma_{2}$ that has cyclic edge groups and a vertex group $\Gamma_{v}$ that is a non-abelian limit group. Then $\Gamma_{v} \cap L_{2} \neq\{1\}$.

Proof. In $\mathcal{G}$ we fix an edge $e$ incident to the vertex $v$ and denote the corresponding edge group $\Gamma_{e} \subset \Gamma_{2}$. We also fix a graph-of-groups decomposition of $\Gamma_{1}$ that has a cyclic edge group $\Gamma_{f}$ (see Proposition 2.1 and the remark following it).

Using Theorem 2.4, we may replace $\Gamma_{1}$ and $\Gamma_{2}$ by subgroups of finite index and assume that they split as HNN extensions with amalgamated subgroups $\Gamma_{e}, \Gamma_{f}$ and stable letters in $L_{1}$ and $L_{2}$, respectively. The Double Coset Lemma (Theorem 2.5) then tells us that $H_{2}(S, \mathbb{Q})$ contains a subgroup isomorphic to

$$
\mathbb{Q}\left(S \backslash\left(\Gamma_{1} \times \Gamma_{2}\right) /\left(\Gamma_{f} \times \Gamma_{e}\right)\right) .
$$

Since $H_{2}(S, \mathbb{Q})$ is finitely generated, $\left|S \backslash\left(\Gamma_{1} \times \Gamma_{2}\right) /\left(\Gamma_{f} \times \Gamma_{e}\right)\right|<\infty$. So, by Proposition 2.6, $\left|\left(S \cap\left(\Gamma_{f} \times \Gamma_{2}\right)\right) \backslash\left(\Gamma_{f} \times \Gamma_{2}\right) /\left(\Gamma_{f} \times \Gamma_{e}\right)\right|<\infty$. Applying the projection $p_{2}: S \rightarrow \Gamma_{2}$, we have $\left|p_{2}\left(S \cap\left(\Gamma_{f} \times \Gamma_{2}\right)\right) \backslash \Gamma_{2} / \Gamma_{e}\right|<\infty$, by Proposition 2.6. Hence

$$
\left|\Gamma_{v} \cap p_{2}\left(S \cap\left(\Gamma_{f} \times \Gamma_{2}\right)\right) \backslash \Gamma_{v} / \Gamma_{e}\right|<\infty,
$$

again by Proposition 2.6. Now, $S \cap\left(\Gamma_{f} \times \Gamma_{2}\right)$ is generated by $L_{2}=p_{2}\left(L_{2}\right)$ and a cyclic group, so if $\Gamma_{v} \cap L_{2}=\{1\}$ then $C=\Gamma_{v} \cap p_{2}\left(S \cap\left(\Gamma_{f} \times \Gamma_{2}\right)\right)$ would be a cyclic group.

But if $C$ were cyclic, then in the non-abelian, residually-free group $\Gamma_{v}$ we would have two cyclic subgroups, $C$ and $\Gamma_{e}$, such that $\left|C \backslash \Gamma_{v} / \Gamma_{e}\right|<\infty$. But this is impossible, for there is an epimorphism, $\phi$ say, from $\Gamma_{v}$ onto a non-abelian free group $F$, where the conclusion (from Proposition 2.6) that the cyclic subgroups $\phi(C)$ and $\phi\left(\Gamma_{e}\right)$ satisfy $\left|\phi(C) \backslash F / \phi\left(\Gamma_{e}\right)\right|<\infty$ is absurd. 
4.4. Completing the proof of Theorem 1.1. We have non-abelian limit groups $\Gamma_{1}, \Gamma_{2}$ and a subdirect product $S \subset \Gamma_{1} \times \Gamma_{2}$ of type $\mathrm{FP}_{2}(\mathbb{Q})$ such that $L_{1}=S \cap \Gamma_{1}$ and $L_{2}=S \cap \Gamma_{2}$ are non-trivial. We want to prove that $S$ has finite index in $\Gamma_{1} \times \Gamma_{2}$. We shall do so by induction on

$$
h:=\operatorname{height}\left(\Gamma_{1}\right)+\operatorname{height}\left(\Gamma_{2}\right),
$$

where height is defined as in subsection 2.1. Let $h_{i}=\operatorname{height}\left(\Gamma_{\mathrm{i}}\right)$ for $i=1,2$.

Corollaries 4.7 and 4.8 cover the case where $h_{2}=0$ and the case where $\Gamma_{2}$ is freely decomposable. Thus, by Proposition 2.1, we may assume that $\Gamma_{2}$ has a graph-ofgroups decomposition $\Gamma_{2} \cong \pi_{1}(\mathcal{G}, X)$ with infinite-cyclic edge groups such that each edge is incident at a vertex where the vertex group is a non-abelian limit group of height $\leq h_{2}-1$. Let $\Gamma_{v}$ be such a vertex group and let $\Gamma_{e}$ be an incident edge group. Lemma 4.9 tells us that $\Gamma_{v} \cap L_{2} \neq\{1\}$. Corollary 4.6 tells us that $S$ has finite index in $\Gamma_{1} \times \Gamma_{2}$ unless $\Gamma_{e} \cap L_{2}=\{1\}$. Thus we proceed with the assumption that $\Gamma_{e} \cap L_{2}=\{1\}$ for all edges $e$ in the graph $X$. (This will ultimately lead to a contradiction.)

Let $p_{2}: S \rightarrow \Gamma_{2}$ be the natural projection and let $S_{e}=p_{2}^{-1}\left(\Gamma_{e}\right)$.

Claim: $S_{e}$ is a limit group.

Since $\Gamma_{e} \cap L_{2}$ is trivial, the projection $S \rightarrow \Gamma_{1}$, which has kernel $L_{2}$, restricts to an injection on $S_{e}$. Thus $S_{e}$ is isomorphic to a subgroup of a limit group and the claim will follow from Theorem 2.2 if we prove that $H_{1}\left(S_{e}, \mathbb{Q}\right)$ is finite dimensional.

By Theorem 2.4, we may assume that $\Gamma_{2}$ splits as an HNN extension with amalgamated subgroup $\Gamma_{e}$ and stable letter $t \in L_{2}$. (This reduction involves passing to a subgroup of finite index $S_{e}^{\prime} \subset S_{e}$, but if $H_{1}\left(S_{e}^{\prime}, \mathbb{Q}\right)$ is finite dimensional, then $H_{1}\left(S_{e}, \mathbb{Q}\right)$ is.)

Let $M=H_{1}\left(L_{1}, \mathbb{Q}\right)$. Analysing the LHS spectral sequence for

$$
1 \rightarrow L_{1} \rightarrow S \stackrel{p_{2}}{\rightarrow} \Gamma_{2} \rightarrow 1
$$

exactly as in the proof of Proposition 4.4, we see that since $H_{2}(S, \mathbb{Q})$ is finite dimensional, $H_{1}\left(\Gamma_{2}, M\right)$ is finite dimensional, and hence so is $H_{0}\left(\Gamma_{e}, M\right)$, by Lemma 3.1 .

We can now calculate $H_{1}\left(S_{e}, \mathbb{Q}\right)$ using the LHS spectral sequence for

$$
1 \rightarrow L_{1} \rightarrow S_{e} \stackrel{p_{2}}{\rightarrow} \Gamma_{e} \rightarrow 1 .
$$

The relevant terms on the $E^{2}$ page are $H_{1}\left(\Gamma_{e}, H_{0}\left(L_{1}, \mathbb{Q}\right)\right)=H_{1}\left(\Gamma_{e}, \mathbb{Q}\right) \cong \mathbb{Q}$ and $H_{0}\left(\Gamma_{e}, M\right)$, which we have just argued is finite dimensional. Thus $H_{1}\left(S_{e}, \mathbb{Q}\right)$ is finite dimensional and our claim is proved.

The graph of groups decomposition $\Gamma_{2} \cong \pi_{1}(\mathcal{G}, X)$ pulls back to a graph of groups decomposition $S \cong \pi_{1}(\mathcal{S}, X)$ with the same underlying graph $X$. Here the vertex and edge groups have the form $S_{v}=p_{2}^{-1}\left(\Gamma_{v}\right)$ and $S_{e}=p_{2}^{-1}\left(\Gamma_{e}\right)$ respectively, as $v$ and $e$ vary over the vertices and edges of $X$. We have just shown that the edge groups $S_{e}$ are all limit groups. In particular each $S_{e}$ has a finite classifying space [1], and so is of type $\mathrm{FP}_{\infty}(\mathbb{Q})$.

We are assuming that $S$ is of type $\mathrm{FP}_{2}(\mathbb{Q})$, so by Lemma 3.2, we can now deduce that the vertex groups $S_{v}$ are all of type $\mathrm{FP}_{2}(\mathbb{Q})$.

For the remainder of the proof we focus our attention on a single non-abelian vertex group $V:=\Gamma_{v}$ in $\mathcal{G}$ and the corresponding vertex group $S_{v}$ in $\mathcal{S}$. Let $\Lambda$ be the projection of $S_{v}$ to $\Gamma_{1}$. Since $H_{1}\left(S_{v}, \mathbb{Q}\right)$ is finite dimensional, so is $H_{1}(\Lambda, \mathbb{Q})$. By 
Theorem 2.2, $\Lambda$ is a limit group (of height at most $h_{1}$ ). Moreover, $V$ is a limit group of height strictly less than $h_{2}$ (see 2.1).

Thus we have a product of limit groups $\Lambda \times V$ with the sum of heights $<h$, and a subgroup $S_{v} \subset \Lambda \times V$ that is of type $\mathrm{FP}_{2}(\mathbb{Q})$ and projects onto each factor. Moreover $S_{v} \cap \Lambda=L_{1}$, which is non-abelian since it is non-trivial and normal in the non-abelian limit group $\Gamma_{1}$. Hence, in particular, $\Lambda$ is non-abelian.

We also have, by hypothesis, that $V$ is non-abelian and that $V \cap L_{2} \neq\{1\}$. Thus we may apply our inductive hypothesis to conclude that $S_{v}$ has finite index in $\Lambda \times V$. Since $L_{1}=S_{v} \cap \Lambda$, we conclude that $L_{1}$ has finite index in $\Lambda$ and hence is finitely generated. Lemma 4.3 then implies that $S$ has finite index in $\Gamma_{1} \times \Gamma_{2}$. This contradicts the fact that $S \cap \Gamma_{e}$ was assumed to be trivial for each of the infinite-cyclic edge groups $\Gamma_{e} \subset \Gamma_{2}$.

\section{Abelian factors}

Our main theorem concerns a subdirect product of two non-abelian limit groups. The result is false as stated in the case where one or both of the limit groups is allowed to be abelian.

An abelian limit group is just a free abelian group of finite rank. In particular, every subgroup of a direct product of two such groups is of type $\mathrm{FP}_{\infty}(\mathbb{Q})$. More generally, one can easily construct counterexamples to the exact analogue of the main theorem in which one of the factors is allowed to be non-abelian, along the following lines.

Let $\Gamma_{1}$ be a non-abelian limit group, and $f_{1}: \Gamma_{1} \rightarrow \mathbb{Z}^{k}$ an epimorphism for some $k \geq 1$. Let $\Gamma_{2}=\mathbb{Z}^{m}$ for some $m>k$, and let $f_{2}: \Gamma_{2} \rightarrow \mathbb{Z}^{k}$ be an epimorphism. Let $S \subset \Gamma_{1} \times \Gamma_{2}$ be the fibre product of $f_{1}$ and $f_{2}$. Then $S \cong \Gamma_{1} \times \mathbb{Z}^{m-k}$ is a subdirect product of type $\mathrm{FP}_{\infty}$ and $S \cap \Gamma_{i}$ is non-trivial for $i=1,2$, but $S$ does not have finite index in $\Gamma_{1} \times \Gamma_{2}$.

It is not difficult to show that all counterexamples to the exact analogue of the main theorem are, up to finite index, isomorphic to one of the above types. In particular, in each case the group $S$ under consideration is itself a direct product of limit groups, up to finite index.

This observation is a special case of Corollary 1.3. We are now ready to prove Corollary 1.3 , which we restate here as

Corollary 5.1. Let $\Gamma_{1}, \ldots, \Gamma_{n}$ be limit groups, with $\Gamma_{3}, \ldots, \Gamma_{n}$ abelian, and let $S \subset$ $\Gamma_{1} \times \cdots \times \Gamma_{n}$ be a subdirect product such that $S$ is of type $\mathrm{FP}_{m}(\mathbb{Q})$ (where $m=$ $\min (n, 3))$. Then $S$ is isomorphic to a subgroup of finite index in a product of at most $m$ limit groups.

Proof. If all the $\Gamma_{i}$ are abelian, then $S$ is a free abelian group of finite rank, and hence a limit group. If, on the other hand, none of the $\Gamma_{i}$ are abelian, then the result follows from Corollary 1.2. Hence we may assume that $n \geq 2$, that $\Gamma_{1}$ is non-abelian, and that $\Gamma_{n}$ is abelian.

If $\Gamma_{n-1}$ is abelian, let $G_{n-1}$ be the image of $S$ under the direct projection $\Gamma_{1} \times \cdots \times$ $\Gamma_{n} \rightarrow \Gamma_{n-1} \times \Gamma_{n}$. The result for $S \subset \Gamma_{1}, \ldots, \Gamma_{n}$ follows easily from the corresponding result for $S \subset \Gamma_{1} \times \cdots \times \Gamma_{n-2} \times G_{n-1}$.

Hence we may assume that there is precisely one abelian factor, namely $\Gamma_{n}$, and hence that $n \in\{2,3\}$. Let $L_{n}=S \cap \Gamma_{n}$. Since $\Gamma_{n}$ is abelian, we may assume (up 
to replacing $\Gamma_{n}$ by a finite index subgroup) that $L_{n}$ is a direct factor of $\Gamma_{n}$. Say $\Gamma_{n} \cong L_{n} \oplus M$.

Let $\pi: \Gamma_{n} \rightarrow L_{n}$ be the projection with kernel $M$, and define $\theta:=\pi \circ p_{n}$ : $\Gamma_{1} \times \cdots \times \Gamma_{n} \rightarrow L_{n}$. Then $\left.\theta\right|_{S}$ is a splitting of the inclusion map $L_{n} \rightarrow S$, so $S \cong T \times L_{n}$, where $T=\operatorname{Ker}\left(\left.\theta\right|_{S}\right)=S \cap\left(\Gamma_{1} \times \cdots \times \Gamma_{n-1} \times M\right)$.

Now $T \cap M=\{1\}$, and so $T$ is isomorphic to a subgroup of $\Gamma_{1} \times \cdots \times \Gamma_{n-1}$. Moreover, $T$ is of type $\mathrm{FP}_{m}(\mathbb{Q})$ since $S \cong T \times L_{n}$ is of type $\mathrm{FP}_{m}(\mathbb{Q})$. It follows from the main theorem that $T$ has finite index in $\Gamma_{1} \times \cdots \times \Gamma_{n-1}$.

Hence $S$ is, up to finite index, a product of at most $m$ limit groups, as claimed.

\section{The natural conjecture for $n$ factors}

The results of this paper, and of [8], encourage us to make the following conjecture, which would give a positive answer to a question of Sela [19].

Conjecture 6.1. Let $\Gamma_{1}, \ldots, \Gamma_{n}$ be limit groups, and $S$ a subgroup of $\Gamma_{1} \times \cdots \times \Gamma_{n}$ such that $S$ has type $\mathrm{FP}_{n}(\mathbb{Q})$. Then $S$ has a subgroup of finite index that is itself a direct product of $n$ or fewer limit groups.

More precisely, we believe:

Conjecture 6.2. Let $\Gamma_{1}, \ldots, \Gamma_{n}$ be non-abelian limit groups and $S \subseteq \Gamma_{1} \times \cdots \times \Gamma_{n}$ a subdirect product that intersects each $\Gamma_{i}$ non-trivially. If exactly $k>0$ of the intersections $S \cap \Gamma_{i}$ are not finitely generated, then there exists a subgroup of finite index $S_{0} \subset S$ such that $H_{k}\left(S_{0}, \mathbb{Q}\right)$ is infinite dimensional.

The arguments in [7] and [8] prove these conjectures in the case where the $\Gamma_{i}$ are subgroups of elementarily free groups. Those arguments used in a crucial way the fact that the top of the $\omega$-residually free tower decomposition is a quadratic block. It does not seem plausible to extend the methods of [8] to the case where each of the $\Gamma_{i}$ has an abelian block at the top of its $\omega$-rft decomposition.

In the present paper, we addressed the case where at most 2 of the $\Gamma_{i}$ are nonabelian, which reduces easily to the case of precisely two factors, each non-abelian. This more algebraic approach (which is closer to the argument we used with Miller and Short in the surface case [6]) may eventually lead to a proof in the general case. However, there are considerable difficulties associated to the fact that one must repeatedly pass to subgroups of finite index.

Since the work for the present paper was done, we have made significant progress on the above conjectures in joint work with Chuck Miller and Hamish Short. This will be the subject of a forthcoming paper.

\section{Acknowledgements}

This work was supported in part by Franco-British Alliance project PN 05.004. The first author is also supported by an EPSRC Senior Fellowship and a Royal Society Wolfson Research Merit Award. 


\section{References}

[1] E. Alibegović and M. Bestvina, Limit groups are CAT(0), J. London Math. Soc. (2) 74 (2006) 259-272.

[2] B. Baumslag, Residually free groups, Proc. London Math. Soc. (3) 17 (1967), no. 1, 402-418.

[3] G. Baumslag and J. E. Roseblade, Subgroups of direct products of free groups, J. London Math. Soc. (2) 30 (1984), no. 1, 44-52.

[4] M. Bestvina and M. Feighn, Notes on Sela's work: Limit groups and Makanin-Razborov diagrams, in M. R. Bridson, P. H. Kropholler, and I. J. Leary, editors, Geometry and Cohomology in Group Theory (Durham, 2003) (to appear).

[5] R. Bieri, Homological dimension of discrete groups, Queen Mary College Mathematics Notes (1976).

[6] M. Bridson, J. Howie, C. F. Miller III, and H. Short, The subgroups of direct products of surface groups, Geometriae Dedicata 92 (2002) 95-103.

[7] M. R. Bridson and J. Howie, Normalizers in limit groups, Math. Ann. 337 (2007) 385-394.

[8] - Subgroups of direct products of elementarily free groups, J. Geom. and Functl. Anal. (to appear)

[9] M. R. Bridson and D. T. Wise, VH-complexes, towers and subgroups of $F \times F$, Math. Proc. Cambridge Philos. Soc. 126 (1999) 481-497.

[10] K. S. Brown, Cohomology of groups, Vol. 87 of Graduate Texts in Mathematics, Springer-Verlag, Berlin-Heidelberg-New York (1994).

[11] C. Champetier and V. Guirardel, Limit groups as limits of free groups, Israel J. Math. 146 (2005) 1-75.

[12] P. J. Hilton and U. Stammbach, A course in homological algebra, Vol. 4 of Graduate Texts in Mathematics, Springer-Verlag, Berlin-Heidelberg-New York (1997).

[13] O. Kharlampovich and A. Myasnikov, Irreducible affine varieties over a free group. I. Irreducibility of quadratic equations and Nullstellensatz, J. Algebra 200 (1998) 472-516.

[14] - Irreducible affine varieties over a free group. II. Systems in triangular quasi-quadratic form and description of residually free groups, J. Algebra 200 (1998) 517-570.

[15] — Elementary theory of free nonabelian groups, J. Algebra 302 (2006) 451-552.

[16] C. F. Miller III, Subgroups of direct products with a free group, Quart. J. Math. 53 (2002) 503-506.

[17] V. N. Remeslennikov, $\exists$-free groups, Sibirsk. Mat. Zh. 30 (1989), no. 6, 193-197.

[18] G. P. Scott, Subgroups of surface groups are almost geometric, J. London Math. Soc. (2) 17 (1978) 555-565.

[19] Z. Sela, Diophantine geometry over groups: a list of research problems, preprint http://www.ma.huji.ac.il/ zlil/problems.dvi.

[20] - Diophantine geometry over groups. I. Makanin-Razborov diagrams, Publ. Math. Inst. Hautes Études Sci. (2001), no. 93, 31-105.

[21] —, Diophantine geometry over groups. II. Completions, closures and formal solutions, Israel J. Math. 134 (2003) 173-254.

[22] - Diophantine geometry over groups VI: The elementary theory of a free group, Geom. Funct. Anal. 16 (2006) 707-730.

[23] H. Short, Finitely presented subgroups of a product of two free groups, Quart. J. Math. 52 (2001) 127-131.

[24] J. R. Stallings, A finitely presented group whose 3-dimensional homology group is not finitely generated, Amer. J. Math. 85 (1963) 541-543.

Department of Mathematics, Imperial College London, London SW7 2AZ

E-mail address: m.bridson@imperial.ac.uk

URL: http://www.ma.ic.ac.uk/ mbrids/

Department of Mathematics, Heriot-Watt University, Edinburgh EH14 4AS

E-mail address: J.Howie@hw.ac.uk

$U R L:$ http://www.ma.hw.ac.uk/ jim/ 